\title{
$H P^{3}$ - Experiment on InSight Mission - Operations on Mars
}

Christian Krause ${ }^{1}$, Cinzia Fantinati ${ }^{1}$, Elizabeth Barret ${ }^{3}$, Matthias Grott ${ }^{2}$, Troy Hudson ${ }^{3}$, Sven Jansen ${ }^{1}$, Judit Jänchen ${ }^{2}$, Joerg Knollenberg ${ }^{2}$, Daniel May ${ }^{1}$, Carolyn Parcheta ${ }^{3}$, Aleksandra Rutcynska ${ }^{1}$, Jaime Singer ${ }^{3}$, Sue Smrekar ${ }^{3}$, Tilman Spohn ${ }^{2}$, Louise Thomas ${ }^{1}$, Markus Wiedemann ${ }^{4}$

${ }^{1}$ DLR Microgravity User Support Center (MUSC), Cologne, Germany

${ }^{2}$ DLR Institute of Planetary Research, Berlin, Germany

${ }^{3}$ Jet Propulsion Laboratory, California Institute of Technology, Pasadena, USA

${ }^{4}$ Magson $\mathrm{GmbH}$, Berlin, Germany

$\mathrm{HP}^{3}$ - the Heat Flow and Physical Properties Package - is an experiment package on-board the upcoming NASA Mars Mission InSight (Interior Exploration Using Seismic Investigation, Geodesy, and Heat Transport).The InSight Mission will investigate the interior structure of Mars using seismoligical and geodetical measurements and quantify the planetary heat budget by measuring the surface planetary heat flow. InSight is scheduled to be launched in May 2018 and to land on Mars at end of November 2018. The main payloads of the InSight lander are a seismometer (SEIS), the $\mathrm{HP}^{3}$ heat flow probe, as well as the Rotation and Interior Structure Experiment (RISE). An ancillary sensor package consisting of atmospheric pressure and temperature sensors (APSS) as well as a magnetometer complement the payload. After landing on Mars the seismometer and $\mathrm{HP}^{3}$ will be deployed onto the Martian surface by the robotic arm of the lander.

$\mathrm{HP}^{3}$ is the contribution of DLR (Deutsches zentrum für Luft und Raumfahrt, Germany) to the InSight mission. It is designed to determine the geothermal heat flux by penetrating down into the Martian surface to at least $3 \mathrm{~m}$, with the goal of reaching $5 \mathrm{~m}$ depth. $\mathrm{HP}^{3}$ measures the thermal conductivity as function of depth during the penetration phase, and the thermal profile of the subsurface will be monitored for a full Martian year after reaching the final depth.

$\mathrm{HP}^{3}$ is composed of the following subsystems:

- A set of thermal sensors to determine thermal conductivity and subsurface temperature (TEM)

- A self-penetrating probe (termed the mole) to emplace sensors in the subsurface

- Two measurements suites to determine the depth of the thermal sensors (TLM \& STATIL)

- A radiometer to determine the surface temperature forcing (RAD)

- The instrument main (backend) electronics (BEE)

The $\mathrm{HP}^{3}$ deployable elements are housed inside a support structure, and electrical connections to the lander and $\mathrm{BEE}$ are provided by the $\mathrm{HP}^{3}$ supply tethers. The support structure also guides the mole during its initial penetration into the surface. The mole is a mechanically actuated force hammering device for soil penetration. It pulls a tether with thermal sensors and supply lines behind. By penetrating into the Martian subsurface the thermal sensors (TEM) will be deployed. The mole is equipped in its back part with the subsystem STATIL (STATIC Tilt Measurement Suite) which determines the mole inclination during the penetration. The length of paid-out tether is measured by the TLM subsystem (Tether Length Monitor). The path of the mole and the depth of the thermal sensors will be deterimed from the TLM and STATIL data. The radiometer is mounted 
underneath the InSight lander deck and determines the surface brightness temperature of the martian regolith, from which regolith thermal inertia of the the upper soil layers can be derived. The radiometer will perform its measurements on dedicated durations throughout the Martian day and night.The instrument is operated and controled by the $\mathrm{HP}^{3}$ Backend Electronics, which also provides the telemetry and commanding interface to the InSight lander. The $\mathrm{HP}^{3}$ backend electronics is located inside the lander's warm compartement.

The operations of $\mathrm{HP}^{3}$ on Mars will start shortly after landing. First checkouts and first scientific measurements will be performed during the early phases of the InSight surface mission. The first part of the mission is further dominated by the selection of the instrument deployment sites, followed by the deployment of SEIS and $\mathrm{HP}^{3}$ itself onto the surface.

After $\mathrm{HP}^{3}$ is deployed onto the Martian surface the penetration phase will start. During this phase the mole will penetrate stepwise into the subsurface, and stops every $50 \mathrm{~cm}$ are foreseen. At each depth $\mathrm{HP}^{3}$ will perform thermal measurements to determine the thermal conductivity of the regolith, thus providing a thermal conductivity depth profile upon full penetration. After reaching its target depth, mole hammering will stop and the monitoring phase of $\mathrm{HP}^{3}$ will start. This phase will last till the end of mission and is composed of thermal monitoring of the subsurface temperatures als well as radiometer measurements of the Martian surface.

The presentation will provide an introduction to the $\mathrm{HP}^{3}$ experiment and its status, and will focus on the upcoming operations after landing on Mars in late November 2018. 